\title{
Correlating Nanoscale Defects with Electronic Properties in MgO Magnetic Tunnel Junctions by Aberration-Corrected STEM-EELS
}

\author{
P.Y. Huang,* J.J. Cha, *** J.C. Read, **** H-W. Tseng, * Y. Li,* P.G. Gowtham,* \\ R.A. Buhrman*, D.A. Muller* \\ * Applied and Engineering Physics, Cornell University, Ithaca, NY 14853 \\ ** Materials Science and Engineering, Stanford University, Stanford, CA 94305 \\ *** National Institute of Standards and Technology, Gaithersburg, MD 20899
}

Utilizing electron energy loss spectroscopy (EELS) for spectrum imaging (SI) in a fifth-order aberration-corrected scanning transmission electron microscope (STEM), we have observed and characterized chemical diffusion and the formation of nanoscale inclusions in $\mathrm{MgO}$ magnetic tunnel junctions (MTJs). Correlating these effects with electronic characteristics of the films reveals complex and sometimes counter-intuitive relationships between the atomic-scale chemistry and device performance. For instance, our data show that tunneling magnetoresistance (TMR) in films made from sputter-deposited $\mathrm{MgO}$ is quite resilient to interlayer diffusion into the $\mathrm{MgO}$ tunnel barrier and the ferromagnetic electrodes, achieving a high 229\% tunneling magnetoresistance despite nanoscale inclusions and other "defects." Indeed, not all of the diffusive effects we observe may be detrimental to the TMR; for example, our data suggest that boron diffusion into the MgO layer may be beneficial because it blocks more harmful elements such as Fe from migrating into the tunnel barrier. These results have important implications both for designing MTJs and for characterizing them: because of the small scale of diffusion, lateral non-uniformity in the films, and the added complications of behaviors such as metallic oxidation, we demonstrate that a $2 \mathrm{D}$ or higher structural and chemical characterization technique with atomic-scale resolution, such as EELS SI, is key to understanding and engineering these complex materials.

EELS is an ideal technique with which to examine MTJs because not only can EELS SI produce 2D chemical maps at atomic scale [1], but we can also employ multivariate curve resolution (MCR) to separate EEL-spectra based on chemical bonding of elements-allowing us, for example, to distinguish between iron and iron-oxide. Together, EELS and STEM imaging enable us to simultaneously identify, record the crystallization of, and quantify the chemical species present in an MTJ_all at atomic scale.

MgO-based MTJs exhibit large TMR, making them an excellent candidate for both magnetic random access memory (MRAM) and read-head sensor applications. While theoretical predictions of over $1000 \%$ TMR have been made for epitaxial $\mathrm{Fe} / \mathrm{MgO} / \mathrm{Fe}$ tunnel junctions, experiments have been unable to achieve such high TMR in epitaxially grown structures $[2,3]$. Instead, the highest TMR to date, of over $600 \%$ at room temperature, has been reached by $\mathrm{rf}$ sputter-deposition of $\mathrm{MgO}$ with boron-alloyed electrodes [4]. The high TMR observed in sputtered $\mathrm{CoFeB} / \mathrm{MgO} / \mathrm{CoFeB} \mathrm{MTJ}$, in spite of the disorder in these structures, has been attributed to the role of boron diffusion in templating the junction interfaces into their theoretically desired, lattice-matched orientations $[5,6]$. References [7-10] investigate the role of boron in greater detail, demonstrating that $\mathrm{MgO}$ barriers containing boron can yield MTJs with relatively high TMR. Further studies indicate that characterizing diffusion in $\mathrm{MgO}$ is complex because of local variations in chemistry [11]. As work progresses toward the ultra-thin $(<1 \mathrm{~nm})$ tunnel junctions required for spin torque MRAM, the role 
of boron in achieving high TMR, together with the challenges of producing uniform, high quality thin films, motivate a detailed investigation of the chemical properties of high quality MTJs.

We examined MTJs both as-grown and after a $350^{\circ} \mathrm{C}$ vacuum anneal with a sample structure of $(\mathrm{Ta}(5) / \mathrm{CuN}(20)) \times 4 / \operatorname{IrMn}(25) / \mathrm{CoFeB}(4) / \mathrm{MgO}(1.6) / \mathrm{CoFeB}(3) / \mathrm{Ta}(8) / \mathrm{Ru}(7)$ (in nm). While the tunnel junction often appears uniform and well-ordered in BF STEM and TEM images, the chemical behavior of the junctions can be quite complex and vary extensively on the nanometer scale. Among the new phenomena we have observed with EELS SI, our data on an annealed MTJ with a $1.6 \mathrm{~nm}$ barrier layer show: $\mathrm{Fe}, \mathrm{Co}$, and $\mathrm{Mn}$ fingers extending into the $\mathrm{MgO}$ tunnel barrier, $\mathrm{Mn}$ and Fe oxide formation at the $\mathrm{CoFeB} / \mathrm{MgO}$ interfaces, and $\sim 1 \mathrm{~nm}$ diameter inclusions in the bottom Fe electrode (see Figure 1). We also observe a high concentration $(\sim 12 \%)$ of $\mathrm{B}$-oxide in the $\mathrm{MgO}$ layer. Because regions with $\mathrm{Fe}$ and $\mathrm{Co}$ fingers in the tunnel barrier correspond to areas low in B-oxide, we conclude that the formation of an $\mathrm{Mg}-\mathrm{B}-\mathrm{O}$ barrier may actively block diffusion of other metals into the barrier, making it a good candidate for the development of MTJs which are resistant to heat and voltage. Despite their ostensibly "bad" microstructure, we emphasize that current-in-plane-tunneling measurements reveal that the annealed layers achieve over 200\% TMR. Such high TMR in the face of extreme deviation from the classic $\mathrm{Fe} / \mathrm{MgO} / \mathrm{Fe}$ system raises the question of what mechanism may be used to explain TMR in $\mathrm{MgO}$ tunnel junctions with B-alloyed electrodes.

\section{References}

[1] D. A. Muller et al., Science 319 (2008) 1073-1076.

[2] W.H. Butler et al., Phys. Rev. B 63 (2001) 054416.

[3] J. Mathon and A. Umerski, Phys. Rev. B 63 (2001) 220403.

[4] S. Ikeda, et al., Appl. Phys. Lett. 93, 082508 (2008).

[5] D. D. Djayaprawira et al., Appl. Phys Lett. 86 (2005) 092502.

[6] S. Yuasa et al., Appl. Phys Lett. 87 (2005) 242503.

[7] J. J. Cha et al., Appl. Phys Lett. 91 (2007) 062516.

[8] J. C. Read et al., Appl. Phys Lett. 94 (2009) 112504.

[9] J. J. Cha et al., Appl. Phys Lett. 95 (2009) 032506.

[10] D.A. Stewart, Nanoletters 10 (2010).

[11] S. Pinitsoontorn et al., Appl. Phys Lett. 93 (2008) 071901.

[12] This research is supported by the NSF through the Cornell MSREC program and by the ARO.
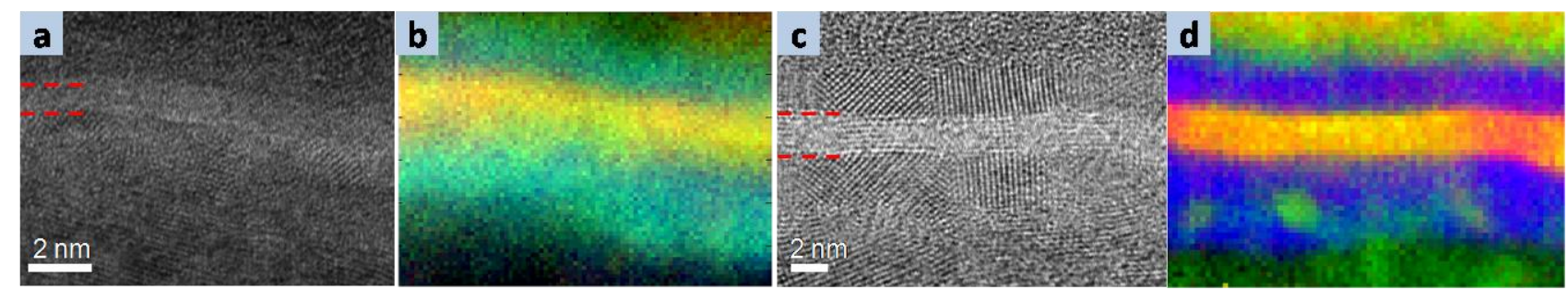

FIG 1. BF STEM and RGB color composition maps of $O, B$, and $\mathrm{Fe}$ in a seed layer/IrMn(25)/CoFeB(4)/MgO(1.6)/CoFeB(3) /capping layer (in nm) thin film stack before $(\mathrm{a}, \mathrm{b})$ and after (c,d) anneal. In each RGB map, $\mathrm{O}$ is displayed in red, $\mathrm{B}$ in green, and Fe in blue, and the $\mathrm{MgO}$ layer is indicated by dashed lines in the BF images. Both before and after anneal, the $\mathrm{MgO}$ layer appears yellow in the RGB maps, indicating that the barrier is an Mg-B-O material. As-grown, the magnetic electrodes appear to be a largely uniform $\mathrm{CoFeB}$ alloy, while after anneal, B leaves the electrodes except in localized inclusions of boron, which appear as green pockets in (d). 